\title{
Valutazione farmacoeconomica di una casistica di pazienti con riacutizzazione di bronchite cronica
}

\author{
M. Liconti, M. Polimeni, c. Filloramo, F. Reale*
}

\section{ABSTRACT}

Aim of this study is to operate a pharmaeconomical evaluation on case histories of patients affected by chronic bronchitis relapse, comparing the effectiveness of different molecules with antibiotic activity and the total cost far each considered drogo.

The observational retrospective study has been conducted at the Bronchopncumology Depaliment of the E. Morelli Hospital (Reggio Calabria) on 344 patients aftècted by acute infective or chronic relapsed bronchopneumopaties between January, the 1st, 1997 and December, the 31th 1999.

To avoid excessive data dispersal, the study considered only the active principles prescribed to at least 50 persons: ceftazidime, ceftriaxone, cefepime and piperacilline + tazobactam.

AlI the patients have responded positively to the treatment, so the cost minimization analysis has been carried out far every dosage regimen, considering different parameters: nursing staff costs, consumable material and drug costs. The Prescribed Daily Dose (PDD) has been taken as unit of measurement for drug costs.

The single adminstration cost is based on nursing costs and consumable material costs. To get the total therapy cost, it's necessary to add the single administration drug costo. In this study, the lowest total cost is guaranteed by treatment with ceftriaxone.

\section{INTRODUZIONE}

Gli episodi di riacutizzazione della bronchite cronica sono definiti come un peggioramento delle condizioni cliniche dei pazienti affetti dalla malattia di base e si manifestano solitamente con un'esacerbazione della tosse e una modificazione dell'escreato che, da mucoso che era, diventa giallo-verdastro se non francamente purulento; può essere presente febbre, in concomitanza con un peggioramento delle condizioni generali. Negli Stati Uniti la bronchite cronica colpisce circa il 25 per cento della popolazione di età superiore ai 40 anni (1), nel 90 per cento dei casi, fumatori.

Anche se qualche volta le riacutizzazioni possono essere correlate a fenomeni allergici o alla presenza di virus o micoplasmi (2), nella stragrande maggioranza dei casi si riconosce una eziologia batterica, confermata anche dall'efficacia del trattamento antibiotico che emerge da diversi studi condotti vs placebo (3). Dal momento che la terapia antibiotica in questi casi viene somministrata sulla base di una scelta empirica, in assenza di una documentazione microbiologica dell' agente infettivo, il medico deve tenere conto della prevalenza di microrganismi quali Streptococcus pneumoniae, Haemophilus influenzae e Moraxella catarrhalis in questo tipo di episodi infettivi $(4,5)$.

Molti studi clinici, condotti anche su ampie casistiche concordano nell'identificazione delle cefalosporine di Il o di 111 generazione quali agenti di prima scelta $(6,7)$.

\footnotetext{
* Ospedale "E. Morelli" di Reggio Calabria
} 
Dal momento che le infezioni delle basse vie respiratorie sono tra le patologie infettive più frequenti tra quelle trattate in regime di ricovero ospedaliero, da alcuni anni ci si pone il problema dell'incidenza del costo della somministrazione di antibiotici a questi pazienti. A tale proposito, per dare un'idea dell'ordine di grandezza dell'impegno economico, vale la pena ricordare che i farmaci antibatterici costituiscono, da soli, il 25-30 per cento circa della spesa ospedaliera complessiva per il compartimento farmaceutico.

Lo scopo di questo studio è di valutare e porre a confronto sia l'efficacia di diverse molecole ad attività antibatterica, che il costo complessivo della terapia somministrata per ognuno dei farmaci presi in esame. Per arrivare alla definizione del costo complessivo è stata considerata una serie di variabili che vanno ben al di là del semplice costo della singola confezione del prodotto.

\section{MATERIALI E METODI}

In questo studio osservazionale retrospettivo condotto presso l'Ospedale E. Morelli di Reggio Calabria sono stati raccolti i dati relativi a tutti i pazienti ricoverati presso uno dei due reparti di Broncopneumologia dal 10 gennaio 1997 al 31 dicembre1999 affetti da bronco-pneumopatie infettive acute o croniche riacutizzate. La raccolta dei dati è stata curata dalla Farmacia Ospedaliera, nell 'intento di accertare non solo l'efficacia dei trattamenti proposti, ma anche l'impatto che questi esercitano sui costi assistenziali della struttura sanitaria. Per questo sono stati registrati in una apposita scheda i dati demografici dei pazienti (età, sesso), il tipo di patologia per la quale sono stati ricoverati, le eventuali patologie concomitanti, l'antibiotico prescritto, l'eventuale associazione con un secondo antibiotico e la sostituzione della terapia, in caso di mancata risposta. Sono stati riportati i dati relativi all'esito del trattamento, distinguendo le risposte complete (guarigioni) dai semplici miglioramenti. Quindi si è passati alla registrazione delle informazioni necessarie alla valutazione farmacoeconomica riportando nel dettaglio, sempre per ogni paziente:

? la quantità in grammi di principio attivo per ogni somministrazione;

? il numero di somministrazioni giornaliere;

? la via di somministrazione utilizzata;

? la durata del trattamento (in giorni).

\section{RISULTATI}

Nel periodo preso in esame i pazienti ricoverati presso uno dei due reparti di Broncopneumologia dell'Ospedale E. Morelli di Reggio Calabria per infezioni delle basse vie respiratorie e sottoposti a terapia antibiotica sono stati 516. Complessivamente sono stati somministrati 17 diversi antibiotici, ma per evitare una eccessiva dispersione dei dati e una scarsa rappresentatività dei risultati ai fini dello studio sono stati presi in considerazione solamente i principi attivi somministrati ad almeno 50 pazienti: si tratta di ceftazidime, ceftriaxone, cefepime e piperacillina + tazobactam. In complesso sono stati considerati 372 pazienti, pari al $72 \%$ del totale dei pazienti trattati. Anche se in nessuno di questi casi è stato necessario modificare la terapia antibiotica, per 13 di essi (ceftazidime $n=8$, ceftriaxone $n=3$, cefepime $n=2$ ) è stata utilizzata una terapia antimicrobica di associazione. Nonostante il basso numero di pazienti trattati in regime politerapico, si è comunque deciso di escluderli dallo studio e di limitare la valutazione clinica e farmacoeconomica ai 359 pazienti trattati in monoterapia con i quattro principi attivi sopra riportati (ceftriaxone $n=116$, ceftazidime $n=112$, cefepime $n=78$ e piperacillina + tazobactam $n=53$ ).

Non sono state osservate differenze significative per quanto riguarda l'età media dei pazienti nei quattro gruppi (rispettivamente 64,0;65,8; 68,7 e 65,5 anni), mentre nel rapporto maschi/femmine si è osservata una prevalenza del sesso femminile tra i pazienti trattati con ceftriaxone e ceftazidime e di quello maschile nei gruppi trattati con cefepime e piperacillina + tazobactam. In 351 casi al momento del ricovero è stata fatta 
diagnosi di bronchite cronica riacutizzata, e per i restanti 8 pazienti risultano varie patologie raggruppabili sotto la denominazione "altre patologie infettive delle basse vie respiratorie".

Dei 359 pazienti trattati in monoterapia, 15 sono stati giudicati non valutabili in quanto nella scheda è risultato mancante l'esito finale della terapia (ceftazidime $n=6$; cefepime $n=4$; piperacillina + tazobactam $n=5)$.

\begin{tabular}{|c|c|}
\hline & Intramuscolare (TM.) \\
\hline Tempo del capo-sala (nimuti) & (range $1-5$ ) \\
\hline Tempo dellinfermiere professionale (minuti) & (range $3-9$ ) \\
\hline Tempo totale (minuti) & 9 \\
\hline Costo per minuto (lire) & 600 \\
\hline Costo del personale (lire) & 5.400 \\
\hline Costo del materiale (lire) & 180 \\
\hline Costo totale di una somministrazione (lire) & 5.580 \\
\hline
\end{tabular}

Tabella 1

Costo di una somministrazione (via iniettiva).

I 344 pazienti considerati valutabili hanno tutti risposto positivamente al trattamento, dimostrando così l'adeguatezza del trattamento attuato. Le differenze evidenziate fra i gruppi terapeutici da un punto di vista clinico sono pertanto in termini di rapporto fra i pazienti guariti e quelli migliorati all'interno di ciascun gruppo e in termini di durata del trattamento. Nel complesso comunque, le differenze di efficacia, calcolata considerando anche i miglioramenti non sono risultate statisticamente significative. I pazienti migliorati venivano dimessi dopo che erano stati tenuti sotto osservazione per un adeguato tempo: tale approccio è stato determinato dalla necessità di un contenimento dei costi ospedali eri, ma anche per ridurre il rischio all'esposizione di patogeni ospedalieri. La durata della terapia è stata in media di 7,64 giomi per ceftriaxone, di 8,97 per ceftazidime, di 9,62 giorni per cefepime e di 10,36 per piperacillina + tazobactam.

\begin{tabular}{|lcccc|}
\hline & ceftriaxone & ceftazidime & cefepime & $\begin{array}{c}\text { piperacillina } \\
+ \text { taz }\end{array}$ \\
\hline $\begin{array}{l}\text { Costo di uma somministrazione IM. } \\
\text { (tempo infernieri + materiale) }\end{array}$ & 5.580 & 5.580 & 5.580 & 5.580 \\
\hline $\begin{array}{l}\text { Durata media della terapia (giorni) } \\
\text { Somministrazionidic (media) }\end{array}$ & 7.64 & 8.97 & 9.62 & 10.36 \\
\hline $\begin{array}{l}\text { Costo complessivo delle } \\
\text { somministrazioni (lire) }\end{array}$ & 1.03 & 2.13 & 1.97 & 1.98 \\
\hline
\end{tabular}

Tabella2

Costo ponderato complessivo del personale e materiale per un ciclo di terapia.

Parallelamente alla valutazione clinica dei trattamenti somministrati è stato effettuato un calcolo dei costi medi per paziente per ognuno dei quattro schemi posologici. La valutazione si è basata su una serie di 
parametri che vanno ben oltre il prezzo di acquisto del farmaco, quali il costo del personale infermieristico e quello dei materiali.

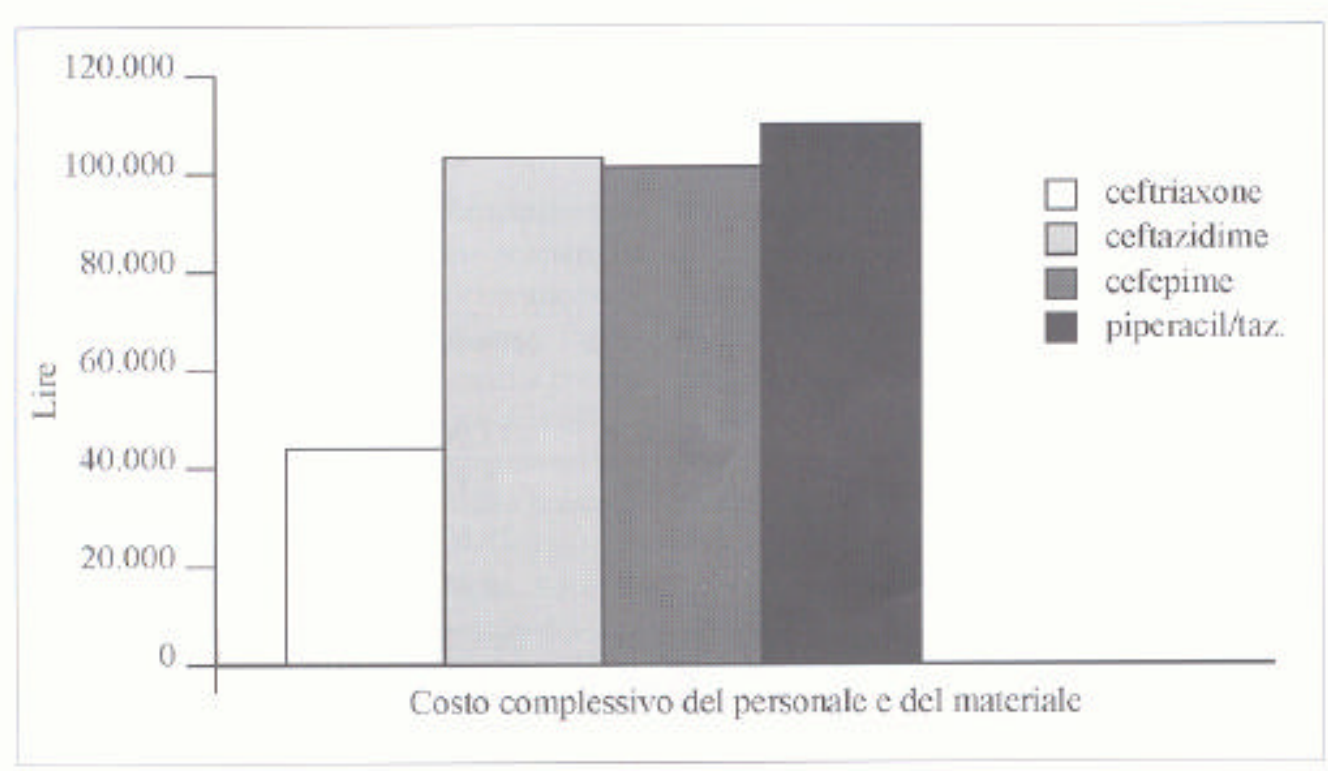

Figura 1

Confronto tra i costi complessivi del personale e del materiale di consumo per un ciclo di terapia con $i$ quattro farmaci in studio.

La prima variabile considerata nel calcolo di costo della terapia è la risorsa "personale" che rappresenta un'entità non trascurabile, di valore variabile rispetto al farmaco, talvolta superiore come importanza a quest'ultimo $(8,9)$.

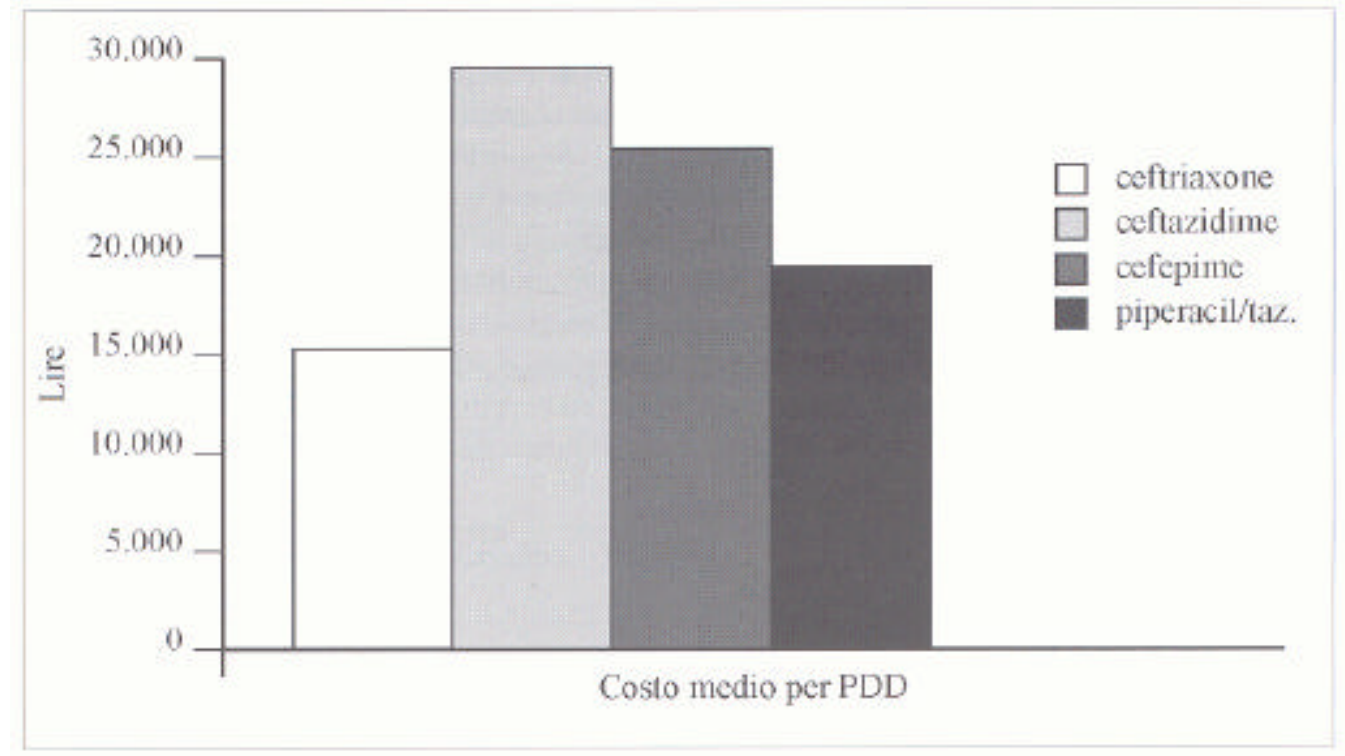

Figura 2

Confronto tra i costi medi per PDD. 
La seconda componente coinvolta nella valutazione dei costi della terapia è quella dei materiali di consumo. Anche il tipo di materiale utilizzato per effettuare le somministrazioni è stato desunto dalla letteratura disponibile(9). Va, inoltre, considerato che da un antibiotico all'altro può cambiare la frequenza di somministrazione (numero di volte al giorno), con conseguenti variazioni dei tempi complessivi e delle quantità di materiale utilizzato.

Per il calcolo del costo per unità di tempo (minuto) sono stati utilizzati i valori monetari descritti dalla letteratura (9,10), riferiti all'anno 1993 e riportati al 1998 tramite gli Indici delle Retribuzioni della Pubblica Amministrazione (11).

Sommando il costo del tempo del personale (capo sala e infermiere professionale) per ogni

somministrazione, al costo dei materiali di consumo (siringa e simili) si è arrivati a definire un costo per ogni singola somministrazione di L. 5.580, uguale per tutti i farmaci in studio essendo la via di somministrazione (iniettiva) uguale per tutti i farmaci (tab.l). Moltiplicando questo costo per la durata media della terapia, che varia da 7,7 giorni per ceftriaxone a IO, 4 per piperacillina + tazobactam, e per il numero medio di somministrazioni giornaliere per ogni farmaco (tab.2), è stato calcolato il costo complessivo del personale e del materiale di consumo per ciclo di terapia (fig. 1). I risultati ottenuti sono suddivisibili in due livelli e precisamente, al livello inferiore ceftriaxone con L. 43.910, e ad un livello più che doppio ceftazidime L. 106.612, cefepime L. 105.749 epiperacillina + tazobactam L.114.461.

Infine è stato calcolato il costo dei farmaci. Per quanto riguarda il consumo di farmaci in termini di quantità sono stati proposti negli anni diversi indicatori $(12,13)$. La dose giornaliera definita (Defined Daily Dose, DDD) è stata proposta come unità di misura ottimale. Essa definisce "lipotetica dose media giornaliera di un farmaco impiegato nel trattamento di un adulto di $70 \mathrm{~kg}$ con riferimento all'indicazione terapeutica principale" (14). Va subito sottolineato però che non si tratta di una media statistica, né di una posologia particolarmente raccomandata, bensì di uno standard di misura. Si evidenzia così il limite della DDD che, proprio in quanto standard universale è poco idonea come misura di consumi effettivi.

Per questo scopo diventa invece necessario uno strumento che, pur avendo una generalità più limitata, sia in compenso più vicino alla contestualità del consumo che si vuole valutare. Tale alternativa è rappresentata dalla dose giornaliera prescritta (Prescribed Daily Dose, PDD). Essa è concettualmente una media statistica; come tale, esprime la tendenza centrale nell'ambito di una variabile rilevata, che nel nostro caso specifico è la dose giornaliera di farmaco mediamente prescritta in un certo ambito ospedaliero.

\begin{tabular}{|lcccc|}
\hline & ceftriaxone & ceftazidime & cefepine & $\begin{array}{c}\text { piperacillina } \\
+ \text { taz }\end{array}$ \\
\hline Costo medio per grammo (lire) & 14.500 & 13.900 & 12.300 & 5.125 \\
\hline Dose giomaliera prescritta (PDD) & 1,04 & 2,13 & 1,97 & 3.58 \\
\hline Costo medio per PDD (lire) & 15.084 & 29.607 & 24.231 & 18.367 \\
\hline Durata media della terapia (giomi) & 7,64 & 8,97 & 9,62 & 10,36 \\
\hline Costo per ciclo di terapia (lire) & 115.244 & 265.575 & 233.102 & 190.282 \\
\hline Costo delle somministrazioni (lire) & 43.910 & 106.612 & 105.749 & 114.461 \\
\hline Costo complessivo della terapia (lire) & 159.155 & 372.187 & 338.851 & 304.743 \\
\hline
\end{tabular}

Tabella3

Costo complessivo della terapia.

Per determinare il costo unitario di ciascuna PDD il primo passo è stato quello di calcolare il costo medio per grammo dei vari farmaci sulla base del prezzo d'acquisto dell'ospedale (50\% circa del prezzo al pubblico). Il costo medio per grammo moltiplicato per la dose giornaliera prescritta (PDD) ha fornito il costo medio per PDD (fig.2); moltiplicando ancora per la durata media della terapia è stato ottenuto il costo per ciclo di terapia. Anche questo valore è risultato molto diverso per i diversi trattamenti somministrati: L. 115.244 per 
ceftriaxone, L. 265.575 per ceftazidime, L. 233.102 per cefepime e L. 190.282 per piperacillina + tazobactam.

Infine sommando questi valori al costo delle somministrazioni calcolato in precedenza è stato identificato il costo complessivo della terapia, specifico per ogni tipo di antibiotico preso in esame. I valori ottenuti sono stati: L. 159.155 per ceftriaxone, L.372.187 per ceftazidime, L. 338.851 per cefepime e L. 304.743 per piperacillina + tazobactam (tab.3 - fig.3).

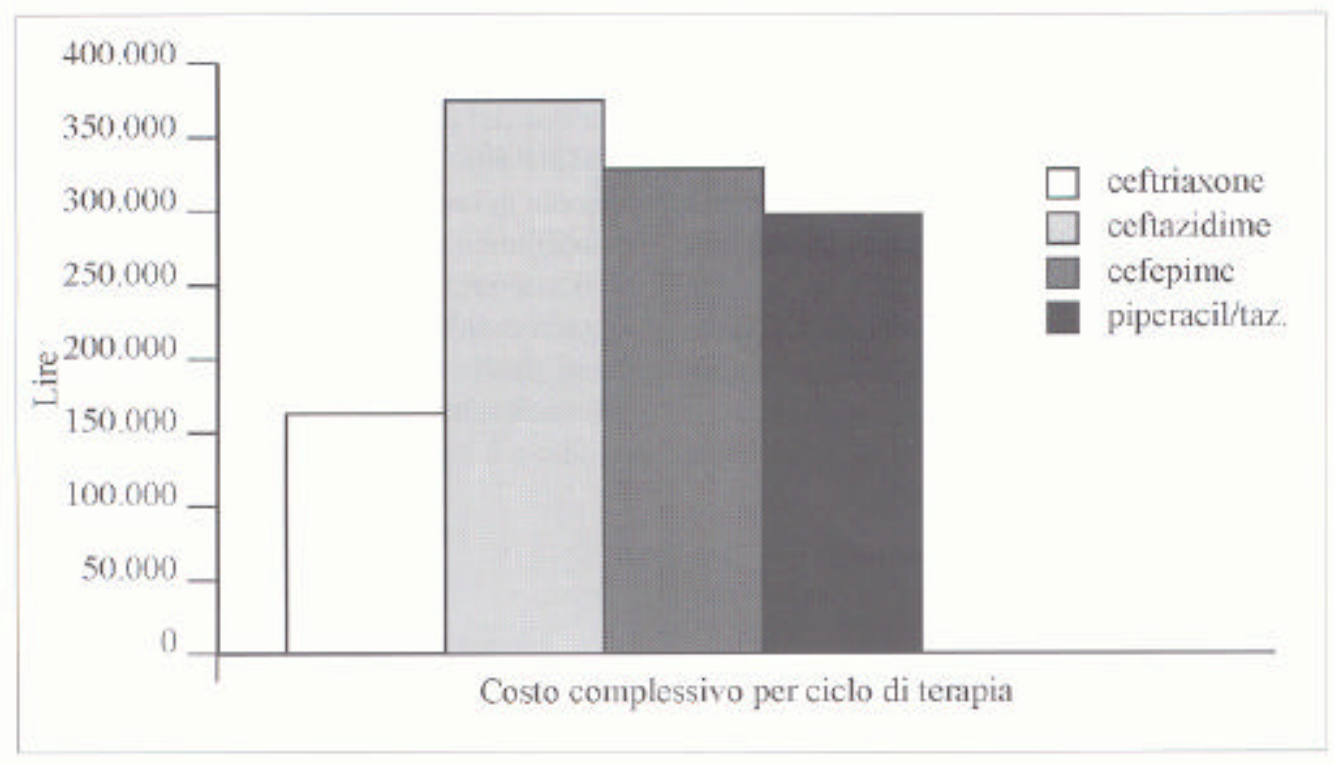

Figura 3

Confronto tra i costi complessivi del trattamento con i quattro farmaci in studio.

\section{DISCUSSIONE E CONCLUSIONI}

L'obiettivo primario dello studio consiste nell'analisi farmacoeconomica dei principali tipi di trattamento antibatterico adottati nei pazienti affetti da riacutizzazione di bronchite cronica. Il primo elemento preso in esame è stato l' efficacia del trattamento. I dati raccolti in questo studio retrospettivo hanno dimostrato l'ottima efficacia dei quattro farmaci valutati e la loro sostanziale equivalenza. La casistica esaminata comprendeva un gruppo importante di pazienti molto anziani, moltissimi con episodi di precedenti ricoveri nello stesso ospedale e per la stessa patologia. Per questo tipo di pazienti l'approccio terapeutico è stato dettato oltre che da considerazioni di efficacia terapeutica anche dalla durata prevedibile del trattamento. Questo parametro ha un impatto importante sia sui costi ospedalieri sia sulla qualità di vita dell'anziano con bronchite cronica. Infatti, quanto più è breve la degenza ospedaliera tanto più è ridotto il rischio di contrarre infezioni ospedaliere da parte di pazienti che spesso presentano un alto numero di patologie concomitanti. Data la sostanziale parità di efficacia dei trattamenti considerati, la valutazione economica si è allora concentrata sul costo dei trattamenti per identificare quello più conveniente. Tecnicamente questa metodologia di analisi prende il nome di analisi di minimizzazione dei costi.

Per ognuno dei farmaci esaminati sono stati registrati: la dose media giornaliera prescritta, il numero medio di somministrazioni giornaliere e la durata media della terapia. La dose giornaliera prescritta (PDD) è una stima del consumo giornaliero pro capite di un certo farmaco in una certa realtà (ospedaliera, ambulatoriale, ecc.) e quindi riflette le abitudini prescrittive in un certo contesto (15). Poiché tiene conto della pratica clinica concreta viene ritenuta più attendibile per una valutazione del dato della dose giornaliera definita 
(DDD), dato teorico fissato a priori e che in quanto standard universale può discostarsi anche significativamente dai consumi effettivi.

Gli altri parametri inclusi nella valutazione del costo complessivo del trattamento sono stati il prezzo per grammo dei singoli farmaci e il costo per le somministrazioni. Quest'ultimo è stato determinato sommando voci diverse, come il costo del caposala e dell'infermiere che effettua la somministrazione nonché il costo del materiale d'uso. Dall'assemblaggio di tutti questi parametri sono emerse differenze interessanti nei profili di costo dei diversi trattamenti. Già per il costo del personale e del materiale d'uso si osserva che a causa delle differenze riscontrate nella durata media del trattamento e nel numero di somministrazioni giornaliere, $\mathrm{i}$ valori che si ottengono vanno dalle 43.910 lire di ceftriaxone alle 114.461 lire di piperacillina + tazobactam. In questo caso sembra giocare un ruolo di primo piano la media delle somministrazioni giornaliere, pari ad 1,03 per il ceftriaxone e compresa tra $1,97 \mathrm{e} 2,13$ per gli altri antibiotici. A questo dato è poi stato aggiunto il costo del farmaco per ciclo di terapia, parametro che si ottiene moltiplicando il costo per grammo di farmaco per la PDD e per la durata media della terapia. Anche tale costo varia significativamente a seconda dell'antibiotico esaminato: si va infatti dalle 115.244 lire di ceftriaxone alle 265.575 lire di ceftazidime. Il costo così alto rilevato per ceftazidime appare correlato al rapporto tra il costo per grammo e la PDD. Infine sommando al costo per il farmaco il costo delle somministrazioni si arriva a definire il costo complessivo della terapia. Ceftriaxone è il farmaco che permette di ridurre al minimo questo costo, che è circa la metà di quello degli altri farmaci presi in esame.

In conclusione, alla luce del peso assunto dagli antibiotici nell'ambito della spesa farmaceutica di una struttura ospedaliera, nonché dell'importanza, a livello epidemiologico, delle patologie infettive a carico delle basse vie respiratorie soprattutto nei pazienti anziani, si ritengono interessanti i risultati qui ottenuti, che trovano peraltro conferma in quanto emerso in studi precedenti (16). In particolare, la marcata riduzione del costo complessivo della terapia osservata con ceftriaxone (rispetto alle altre molecole in studio) induce a considerare questo antibiotico come il farmaco di prima scelta nel trattamento delle riacutizzazioni delle bronchiti croniche, dal momento che la sua efficacia risulta quantomeno sovrapponibile a quella delle altre molecole valutate dal presente studio e il suo costo inferiore.

\section{BIBLIOGRAFIA}

1. Centers for Disease Control. Vital and health interview statistics: current estimates from the National Health lnterview Survey, 1990. US Department of Health and Human Services publication: series 10, No. 181.

2. Buscho RO et al Infections with viruses and Mycoplasma pneumoniae during exacerbations of chronic bronchitis. J Infect Dis 1978: 137: 377-383.

3. Anthonisen NR et al. Antibiotic therapy in exacerbations of chronic obstructive pulmonary disease. Ann Intern Med 1987:106:196-204

4. Mc Fadden RG e Marshall BE. Antibiotic therapy in COPD. Hosp Formul 1992: 27:595-607.

5. Nicotra B et al Branhamella catarrhalis as a lower respiratory tract pathogen in patients with chronic lung disease. Arch Intem Med 1986:146:890-893.

6. McCabe RE et al. Comparison of once daily cephalosporin regimens far community acquired lower respiratory infections in patients with chronic lung disease. Clio Ther 1989: Il: 304-314

7. Maesen FP et al. Ceftriaxone in acute purulent exacerbations of chronic bronchitis. J Antimicrob Chemother 1984:14:653-660

8. Eisemberg J, Glick H, Koffer H. Assessing the hidden cost of antibiotic therapy far hospitalized patients. Drug lnformation J, 22: 459-469,1988.

9. Eandi M, Bondonio PV, Dirindin N. Farmacoeconomia e terapia antibiotica. A.B.E. 1993. 
10. Bondonio PV, Eandi M. Tempi e costi di lavoro associati al trattamento farmacologico: analisi di alcune opportunità di risparmio. Farmaeconomia 2,3: 36-45 1995.

11. ISTAT 1998.

12. Castellani L, Bozzini L, Pedrini A, et al. Prima lista italiana delle DDD di alcuni gruppi di farmaci. Giornale Italiano di Farmacia Clinica 3: 89-116,1989.

13. Lecomte T, Paris V: Consommation de pharmacie en Europe, 1992. CREDES, Paris, 1994.

14. Clark KW, Gray D. The Defined Daily Dose as a tool in Pharmacoeconomics. Advantages and limitations. PharmacoEconomics 7:280-283,1995.

15. Scroccaro G. Farmacoutilizzazione: limiti e applicazioni delle DDD e PDD. Pharmacoeconomics in infectious diseases, 1-7 Aprile 1997.

16. Lucioni C, Concia E, Ravasio R. Terapia empirica con antibiotici parenterali in pazienti affetti da infezioni delle basse vie respiratorie: una valutazione farmacoeconomica. Pharmaco Economics Italian Research Articles. 2001;vol.3,N:1 (pp 37-47). 